\title{
A Dual signal On-Off Fluorescent Nanosensor for the Simultaneous Detection of Copper and Creatinine
}

Resmi V Nair ${ }^{\#}$, Parvathy $R S^{\#}$, Ramapurath S Jayasree*

Sree Chitra Tirunal Institute for Medical Sciences and Technology, Biomedical Technology Wing, Poojappura, Trivandrum

* Correspondence to:

Ramapurath S Jayasree

Division of Biophotonics and Imaging

Biomedical Technology Wing

Sree Chitra Tirunal Institute for Medical Sciences and Technology, Poojappura

Trivandrum

Email: jayasree@sctimst.ac.in, jayashreemenon@gmail.com

Ph:+91 4712520273 , Fax: +91 4712341814

\#These authors contributed equally to this work

\section{Acknowledgements}

The authors acknowledgethe support received from Dr K.K Maiti and his team from CSIRNIIST, Trivandrum and Dr Reji Varghese and his team from IISER, Trivandrum for the TEM studies. Authors also thank Dr. Luxmi Varma R and her team from CSIR-NIIST, Trivandrum for NMR analysis support. Support received from Sreejalekshmi K.G. and Mr. Rakesh from IIST, Trivandrum for fluorescence life time studies is acknowledged.

\section{Declaration of Interest}

Declarations of interest: none 


\title{
Highlights
}

- Green emitting water soluble quantum dots stable at room temperature was prepared

- Meisenheimer complex (PAQd) formation through the functionalization of quantum dot with picric acid through Ethyl-3-(3-dimethylaminopropyl) carbodiimide (EDC) coupling.

- PAQd was used for the simultaneous detection of copper and creatinine and the mechanism of sensing is explored

- Sensing extended to blood serum and cellular environment.

- A paper based strip was developed for the detection and validated it with existing technique using human blood samples.

- Formation of Meisenheimer complex facilitates quick detection at all $\mathrm{pH}$ values

- A paper based strip was developed for the detection and validate it with existing technique using real samples.

\begin{abstract}
The transition of conventional medicine to personalized medicine has paved the road and need for the sensing of newer biomolecules. Hence, this area has attracted wide interest recently, due to its capacity to provide information on point of care basis. Multi-analyte detection sensors have emerged recently as they can offer the opportunity to perform quick and affordable analysis with minimum blood sample, as compared to traditional sensing of each analytes individually. The present study focuses on the development of a quantum dot (Qd) based nanosensor for the simultaneous detection of copper and creatinine; two biologically relevant molecules. The sensor was designed by forming a complex of Qd with Ethyl-3-(3-dimethylaminopropyl) carbodiimide (EDC) and picric acid through carboxylic bond formation of Qd-EDC with picric acid. The dual independent emissions of the Qd-EDC complex was used for the simultaneous detection of creatinine and copper by a turn on/turn off method and was successfully demonstrated with a sensitivity of nanomolar to millimolar, and micromolar to millimolar range respectively. The multianalyte sensor thus developed has
\end{abstract}


quick response and works well under normal conditions of temperature and $\mathrm{pH}$. It is also shown to work in cellular environment and blood serum. A mobile App based detection of creatinine using the developed sensor strips has also been attempted and validated with human blood samples.

Keywords Multianalyte sensor; Analytes; Quantum dots; Fluorescence; Quenching

\section{Introduction}

Nanosensor is one of the strong candidates among the class of both sensors and biosensors with high sensitivity due to the unique properties like high surface to volume ratio of nanostructures. There is an increasing demand for the detection of clinically significant metabolites from body fluids with high selectivity and sensitivity, as an indicator of health status. Currently available methods lack the capability of determining more than one analyte using a single technique from a single draw of blood. Accordingly, the concept of multianalyte sensing has recently been identified as an important need in cases where the information about multiple analytes could be derived using minimum amount of blood in a very short time period. This concept finds importance in cases of diseases having more than one marker for confirmation or more than one diseased condition at the same time. In this context, multi analyte nanosensors (MANS) are the new promising candidates. With the possibility of designing nanostructures with multiple functions, nanosensors are considered to be the most appropriate candidates for simultaneous or multisensing use. However, not many attempts have been made to explore this concept [1-8]. One of the reports among multianalyte sensor deals with quantum dot-DNAzyme based system for multiplexed detection of heavy metals copper and lead ions by using fluorescence property ${ }^{1}$. In another study, the fluorescence property of silica nanoparticles was used for the detection of $\mathrm{Hg}^{2+}$, $\mathrm{H}_{2} \mathrm{SO}^{4-}$ and $\mathrm{S}^{2-i n}$ living cells and for intracellular imaging [2]. Komatsu et al., developed a single fluorescent probe based on coumarin for the detection of multi analytes $\mathrm{Ca}^{2+}$ and $\mathrm{Mg}^{2+}$ ions and for fluorescence imaging [3]. Maria J. Ruedas-Rama and co-workers reported the change in fluorescence property of an enzyme linked nanosphere sensor for the detection of urea and creatinine [4]. In another study, Sugunan et al., worked on chitosan capped gold nanoparticles for the detection of zinc and copper ion by using the chelating property of chitosan along with the optical properties of gold nanoparticles [5]. Among the various nanomaterials, quantum dots have attracted wide acceptance in the designing of optical 
nanosensors for biological and non-biological samples due to its inherent size dependent fluorescence property. Last decade has witnessed revolutionary development of nanoparticle for different biomedical applications [9-21], with Qd leading in the field of nanosensors showing high selectivity and sensitivity compared to conventional sensors.

Herein, we report the development of a two-in-one nanosensor for the simultaneous detection of creatinine and copper using fluorescence emission property of quantum dots, and the mechanism involved.

Biomolecules, creatinine (Creat) and copper $(\mathrm{Cu})$ play a vital role in the metabolism of any living organism. Creat, anhydride form of creatine, is a waste product from the normal breakdown of muscle tissues and is excreted through the kidney without tubular reabsorption. The normal level of creatinine in human blood is 53.041 to $106.082 \mu \mathrm{M}$. Elevated level of creatinine is an indication of impaired renal function, chronic nephritis or urinary tract obstruction and skeletal muscle diseases. Imbalance in creatinine level is an indication of diseases like chronic kidney disease or serious kidney damage, heart failure, dehydration, liver cirrhosis, hyperthyroidism etc [22-24]. Similarly, $\mathrm{Cu}$ is an essential trace element in specific enzymes for proper growth, development and functioning of organs with a normal value of $0.0016-0.0024 \mu \mathrm{M}$ in blood serum. $\mathrm{Cu}$ plays an important role in maintaining and repairing the connective tissues [25]. Decreased levels of copper have been reported in the hearts and arteries of cardiovascular disease patients who are on low copper diet [25]. Deficiency of copper is identified as one of the factors which lead to coronary heart diseases [22]. Other diseases associated with $\mathrm{Cu}$ include nutritional deficiency, inability to absorb copper, Wilson's disease and rheumatoid arthritis [26]. Time bound and early stage detection of these two analytes will give better understanding in maintaining the homeostasis of the body. Though several reports are available for separate detection of copper and creatinine [24,28-36], no studies are available for their simultaneous detection. The simultaneous detection of these molecules is expected to provide immediate and in-depth information on kidney diseases and their related conditions.

\section{Experimental section}

\subsection{Materials}

Cadmium chloride, Selenium powder, sodium sulfite, sodium hydroxide, L-Cysteine, 1Ethyl-3-(3-dimethylaminopropyl) carbodiimide (EDC), copper, creatinine were purchased 
from merck and sigma and used without further purification. MTT powder was purchased from HiMedia. In this paper aqueous medium is DI water.

\subsection{Methods}

\subsubsection{Synthesis of PAQd}

$25 \mathrm{mg}$ of cadmium chloride was treated with $131.505 \mathrm{mg}$ of cysteine and adjust the $\mathrm{pH}$ to 11.5. Then add selenium stock of $125 \mu \mathrm{L}$ (dissolving selenium metal powder to sodium sulfite in $0.2: 0.4 \mathrm{M}$ ratio at $75^{\circ} \mathrm{C}$ for $3 \mathrm{~h}$ in aqueous media) to the solution at $80^{\circ} \mathrm{C}$. The solution was stirred at $590 \mathrm{rpm}$ for $12 \mathrm{~h}$. Qds were purified by centrifugation using isopropanol-water mixture at $10000 \mathrm{rpm}$ for 15 minutes.

Qd was treated with $12 \mathrm{mg}$ of EDC followed by $14 \mathrm{mg}$ of picric acid at normal $\mathrm{pH}$ and 520 rpm stirring for $2 \mathrm{~h}$. The product was separated using centrifugation at $8000 \mathrm{rpm}$ for 10 minutes.

\subsubsection{Characterization of PAQd}

PAQd was characterized using Fourier Transform infrared spectrometer Cary 600 (Agilent Technologies) to confirm the functionalization and chemical changes after the formation of complex with EDC and Qd. IR Spectra were recorded in the range 400 to $3600 \mathrm{~cm}-1$ in transmission mode using $\mathrm{KBr}$ pellet method spanning over 32 scans. NMR analysis was also used to confirm the chemical changes happened in the formation of PAQd. Zeta potential at every step of synthesis was investigated using Malvern Zetasizer NanoZS 90 to investigate the change in charge after functionalization. Surface morphology was confirmed using TEM (100 kv JEM-2010 HiTachi-JEOL, Tokyo, Japan) analysis. UV-Visible spectroscopy was done using Shimadzu UV spectrometer- UV 2600 to confirm the optical property and functionalisation of PAQd. The emission properties of the materials were studied using Varian Carl Eclipse fluorescence spectrophotometer at different excitation wavelengths. All the cell imaging experiments were performed using Olympus IX83 Inverted Fluorescence microscope (Tokyo, Japan) equipped with a cooled CCD camera (XM10, monochrome, Olympus), a metal halide lamp (X-Cite, series 120PC Q), an objective lens (LUCPLFLN 40X PH/0.6, include 10X, Olympus), excitation filters (360-370, 470-495, 540-585), dichroic mirrors (DM410, DM505, DM595), and emission filters (420-460, 510-550, 600IF), controlled with CellSens Imaging software. 


\subsubsection{Sensing of copper and creatinine from aqueous solution}

Different molar concentrations of copper and creatinine in the range from $\mathrm{nM}$ to $\mathrm{mM}$ were prepared. Each was incubated with 1mgmL-1 concentration of PAQd. Then fluorescence reading was noted; for copper detection $531 \mathrm{~nm}$ emissions and for creatinine 440nm emission was utilized.

\subsubsection{Detection of copper and creatinine from blood serum}

$5 \mathrm{ml}$ of human blood (100\% anonymous) was used for the study. The serum was separated and diluted with normal saline and divided into different batches. Each batch was added with different concentrations of copper and creatinine. To this solution, $1 \mathrm{mgmL}-1$ of PAQd was added and fluorescence intensity were noted.

\subsubsection{Cell culture}

One cancer and a normal fibroblast cell lines were chosen for this study: MDA MB-231 (human breast cancer cells) and L929 (mouse alveolar and adipose connective tissue cells) were purchased from ATCC. L929 and MDA MB-231 cells were cultured in Dulbecco's Modified Eagle's Medium supplemented with 10\% (v/v) fetal bovine serum (FBS), 100 $\mu \mathrm{gmL}-1$ penicillin, $100 \mu \mathrm{gmL}-1$ streptomycin and $2.5 \mu \mathrm{gmL}-1$ amphotericin B. All the cell lines were maintained in a humidified atmosphere with $5 \% \mathrm{CO}_{2}$ at $37^{\circ} \mathrm{C}$. All experiments were performed using the following protocol: $85-95 \%$ confluent cells were harvested with trypsin-ethylenediaminetetraacetic acid and sub-cultured in culture dishes at a seeding density of $0.25 \times 10^{6}$ cells per millimeter.

\subsubsection{Cytotoxicity studies}

L929 and MDA MB-231 cells were seeded onto a 96-well plate with cell density of $1 \times 10^{4}$ cells/well. After $24 \mathrm{~h}$ of incubation, the cell were further incubated with fresh media $(100 \mu \mathrm{L}$ per well) containing Qd and PAQd concentrations of 1, 0.5, 0.1, 0.05, 0.01, 0.005 and 0.001 mgmL-1 for $72 \mathrm{~h}$. Then the materials containing media was removed and replaced with 100 $\mu \mathrm{L}$ of fresh DMEM containing 3-(4, 5-dimethylthiazol-2-yl)-2,5-diphenyltetrazolium bromide (MTT-10 $\mu \mathrm{L}, 5 \mathrm{mgmL}-1)$. Following $3 \mathrm{~h}$ of incubation period, the insoluble MTT formazan crystals formed were dissolved with DMSO $(100 \mu \mathrm{L})$. Absorbance at $570 \mathrm{~nm}$ was 
measured using a microplate reader (Synergy H1 hybrid multi-model microplate reader, BioTech). The relative cell viability (\%) for each construct related to the control cells was finally calculated.

\subsubsection{Intra cellular copper and creatinine detection}

L929 and MDA MB-231 cells were seeded in a 4 well plate and allowed to grow in the DMEM medium for $24 \mathrm{~h}$. Each well were incubated with different concentrations of copper $(20 \mu \mathrm{M}$ and $3 \mathrm{mM})$ and creatinine $(3 \mathrm{nM}$ and $0.033 \mathrm{mM})$. After $30 \mathrm{~min}$ of incubation, cell culture medium was removed and fresh medium containing and $0.01 \mathrm{mgmL}-1$ of PAQd (sensor) was added and incubated for $3 \mathrm{~h}$. For the control group, similar procedure was performed without the incubation of analytes. A negative control is used without PAQd incubation (data not shown). For the detection, fluorescence microscopic images were captured with same exposure and gain time. Intensity of the images was calculated using gray scale readings.

\subsubsection{Extra cellular copper and creatinine detection}

L929 and MDA MB-231 cells were seeded in a 4 well plate and allowed to grow in the DMEM medium for $24 \mathrm{~h}$. Then each well were incubated with $0.01 \mathrm{mgmL}-1$ of PAQd for 30 minutes followed by different concentrations of copper (20 $\mu \mathrm{M}$ and $3 \mathrm{mM})$ and creatinine (3 $\mathrm{nM}$ and $0.033 \mathrm{mM}$ ) for 15 minutes. For the control group same cells without the incubation of analytes were used. A negative control was used without PAQd incubation (data not shown). For the detection, fluorescence microscopic images were captured with same exposure and gain time. Intensity of the images was calculated using gray scale readings.

\subsubsection{Mobile App based sensing using paper strip sensors: validation with clinical results}

Senor strips of $5 \mathrm{~cm}$ length and $1 \mathrm{~cm}$ width were made from Whatman filter paper. The strips were coated with $50 \mu \mathrm{l}$ of $1 \mathrm{mg} / \mathrm{ml}$ of PAQd by keeping the strips immersed in it. Different concentrations like $8,19,29,38,47$ and $57 \mu \mathrm{M}$ of creatinine were added to different sensor strips followed by shining them with UV lamp (254nm). Pixel intensity of the mobile camera (20MP) images of these sensor strips were calculated using software imageJ and Adobe Photoshop. To extend the sensing to blinded samples of clinical origin, Blood from four different individuals were collected. $100 \mu \mathrm{L}$ of separated serum was diluted with $2 \mathrm{ml}$ of 
saline. These were used to detect creatinine using the paper strip sensor method. The same blood samples were validated for the values by checking the creatinine level in a certified laboratory using currently adopted clinical method.

\section{Result \& Discussions}

\subsection{Synthesis and characterisation of nanosensor PAQd}

Here, a nanosensor was developed using cysteine capped cadmium selenium quantum dots (Qds). L-cysteine, a water soluble amino acid was used as capping agent considering its ability to form complex with copper. The as synthesized Qds had a transparent red color without any visual aggregation. Pure Qd was isolated by centrifugation at $15000 \mathrm{rpm}$ for 15 min with 1:2 mixture of water and isopropanol. To enable multi-analyte sensing, a sensor should be capable of producing different signals in the presence of desired analytes. It is also important that these signals should not interfere with each other. Hence the designing of such a sensor requires special attention. Here, the Qds were then functionalised with picric acid through 1-Ethyl-3-(3-dimethylaminopropyl) carbodiimide (EDC). EDC serves as a spacer and functional moiety which holds picric acid with Qd to form the multianalyte sensor PAQd. Again, picric acid was specifically chosen as a candidate for creatinine sensing. In a typical Jaffe reaction involving picric acid and creatinine, the sensing is comparatively delayed and takes place only at basic $\mathrm{pH}$. Herein, we try to overcome this limitation with respect to $\mathrm{pH}$ and also quicken the process of sensing in the new multisensor. To facilitate sensing of both analytes, the number of moles of EDC and PA were chosen optimally $\left(2 \times 10^{-4} \mathrm{~mol}\right.$ of cysteine and $1 \times 10^{-4} \mathrm{~mol}$ of EDC and picric acid). The orange colored multianalyte sensor PAQd was purified by centrifugation at $10000 \mathrm{rpm}$ for $10 \mathrm{~min}$ and characterized for its properties.

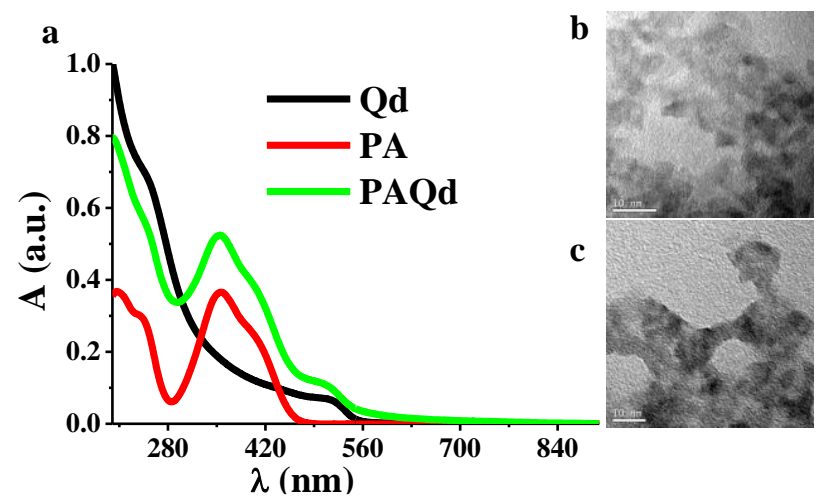

Figure 1. (a) Absorption spectra of Qd, PA and PAQd. PAQd shows characteristic peaks of both PA and Qd (b\&c) Electron Micrographs of Qd and PAQd nm 
In figure 1a, typical absorption peak of bulk cadmium selenide at 698 is absent, ruling out the presence of bulk material [37]. Successful formation of Qd without any aggregation is confirmed by the absorption at $515 \mathrm{~nm}$ (Figure. 1a). Band gap of Qd is found to be $2.41 \mathrm{eV}$ which is at par with the estimated size of $5.4 \pm 0.23 \mathrm{~nm}$ from transmission electron micrograph (Figure 1b). Picric acid (PA) conjugation to Qd resulted in an additional absorption c.a. $354 \mathrm{~nm}$ with a shoulder peak at around $403 \mathrm{~nm}$ (Figure 1a). The peak corresponding to Qd was slightly blue shifted from $515 \mathrm{~nm}$ to $502 \mathrm{~nm}$ due to change in electronic environment effect caused by the conjugation of picric acid- EDC complex over Qd. Qd embedded in the organic matrix is expected to have high energy and is less stable as compared with $\mathrm{Qd}$, and hence is more susceptible to bind with the analytes to attain more stability. The electron micrograph confirms the presence of organic layer around Qd with a size of 5.05 $\pm 0.4 \mathrm{~nm}$ for the complex (Figure 1c). The band gap of Qd increases from 2.41 to $2.47 \mathrm{eV}$ which is an indication of size reduction and is confirmed from the electron micrographs.
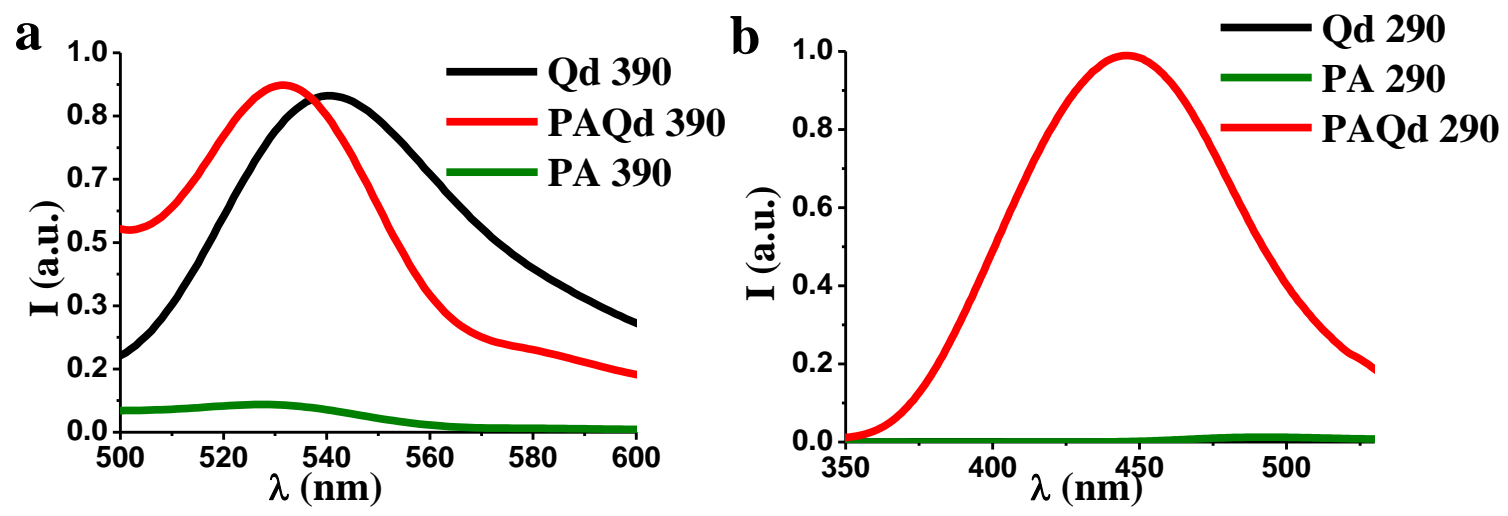

Figure 2. Emission spectra of Qd, PA and PAQd (a) $\lambda_{\text {ex }} 390 \mathrm{~nm}$ (b) $\lambda_{\text {ex }} 290 \mathrm{~nm}$

Qd showed a green emission around $543 \mathrm{~nm}\left(\lambda_{\mathrm{ex}}-360 \mathrm{~nm}\right)$ with a fluorescence life time of $4 \mathrm{~ns}$ (Figure S1 a\&b, Figure S2a) which is blue shifted to $531 \mathrm{~nm}$ on picric acid functionalization (Figure 2a) in agreement with the shift in the absorption peak of Qd. Change in the electron cloud density around Qd due to the presence of EDC-picric acid complex contributed to the shift in the emission profile. The complex Qd-EDC-picric acid (PAQd) showed another prominent emission peak at $446 \mathrm{~nm}$ upon $290 \mathrm{~nm}$ excitation, with fluorescence life time of 5 ns (Figure S2b). This peak is identified as that of the complex as it is neither due to Qd nor 
due to picric acid. Thus, the final nanosensor possesses two independent emissions at two different excitations to support multianalyte sensing (Figure $2 \mathrm{a} \& 2 \mathrm{~b}$ ).

Absence of vibrations of S-H bond of cysteine (around $2561 \mathrm{~cm}^{-1}$ ) in the FT- IR spectrum confirms the formation of S-M (metal) bond in Qd. Broad peak at $3425 \mathrm{~cm}^{-1}$ represents intermolecular hydrogen bonding. The hydroxyl peak of PA at $3425 \mathrm{~cm}^{-1}$ disappears following the functionalization of Qd with EDC and PA, due to the involvement of hydroxyl group, during this step. Presence of aromatic ring is confirmed from the peaks around 3000 $\mathrm{cm}^{-1}$ and $600-900 \mathrm{~cm}^{-1}$ and the nitro group by the peak at $1334 \mathrm{~cm}^{-1}$. Carboxylate group adjacent to nitrogen atom assigned at $1695 \mathrm{~cm}^{-1}$ confirms the complex formation with Qd. FTIR spectral finding suggests that the hydroxyl group of picric acid binds to the active site of Qd-EDC system (Figure. S3). Zeta potential of Qd which was $-16.7 \pm 0.84 \mathrm{mV}$ (Figure. S4) increases to $-14.9 \mathrm{mV}$ for PAQd due to the functionalisation with more of nitro groups of picric acid. $\mathrm{NMR}$ peaks of $\mathrm{NH}, \mathrm{CH}$, and $\mathrm{CH}_{2}$ of $\mathrm{Qd}$ at 1.81, 3.64 and 3.42- $3.52 \mathrm{ppm}$ respectively were visible in the proton NMR spectra (Figure S5a). The peak corresponding to carboxylic hydrogen is not observed due to the formation of intermolecular hydrogen bonding which agrees with the FTIR data. In the NMR spectrum of PAQd, no acidic hydrogen is observed indicating that the phenolic $\mathrm{OH}$ is participating in the formation of the sensor PAQd. The picric acid conjugation is reflected by the presence of aromatic ring in the region, $8.86 \mathrm{ppm}$ and $8.36 \mathrm{ppm}$. All other characteristic peaks of $\mathrm{NH}, \mathrm{CH}$ and $\mathrm{CH}_{2}$ are observed with slight chemical shifts, indicative of different functionalisation of the system (Figure S5b).

\subsection{Mechanism of formation of PAQd}

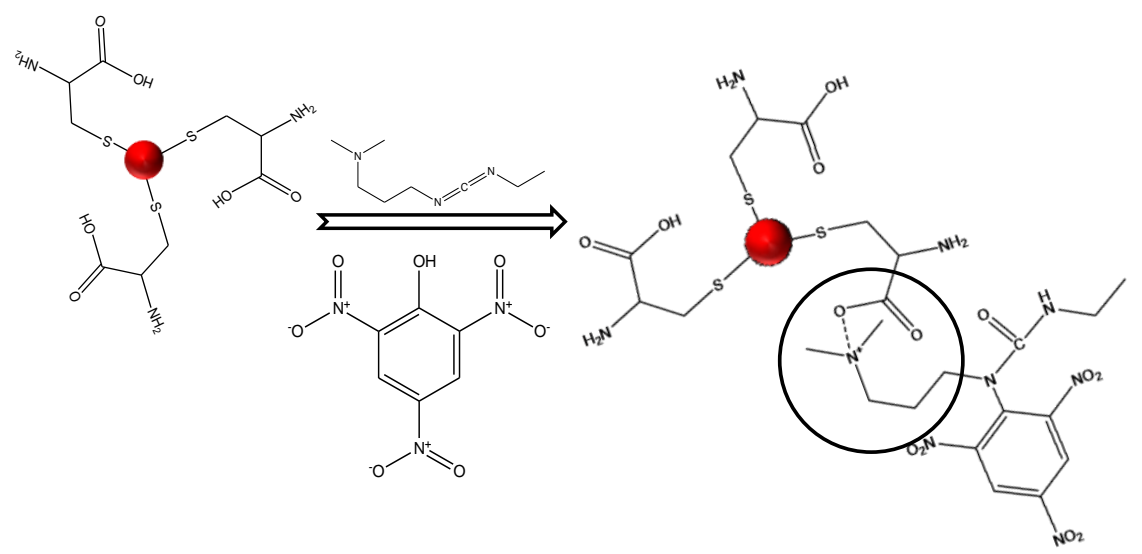

Scheme 1. Schematic representation of formation of PAQd from Qd.Complex formation of PA, EDC and Qd is represented in the circle. 
From the above results, a mechanism of formation of the sensor is proposed. EDC forms an electrostatic bond with carboxylic group $\left(\mathrm{O}^{-}\right)$of cysteine. The diisocarbodiimide group of EDC react with picric acid (PA) resulting in the formation of multifunctional probe (PAQd) [38]. Here, Meisenheimer complex is formed from the reaction of two equivalents of diisopropylcarbodiimide with the substituted phenol, resulting the formation of a substituted s-triazinespirocyclohexadiene ring system [38]. The presence of $1695 \mathrm{~cm}^{-1}$ peak in FTIR spectrum of PAQd confirms the formation of this complex. Due to the presence of Qd it loses zwitter ionic nature by electrostatic interaction to the positive part to negatively charged Qds. Scheme 1 represents the formation of nanosensor.

\subsection{Sensing of copper and creatinine from aqueous solution}

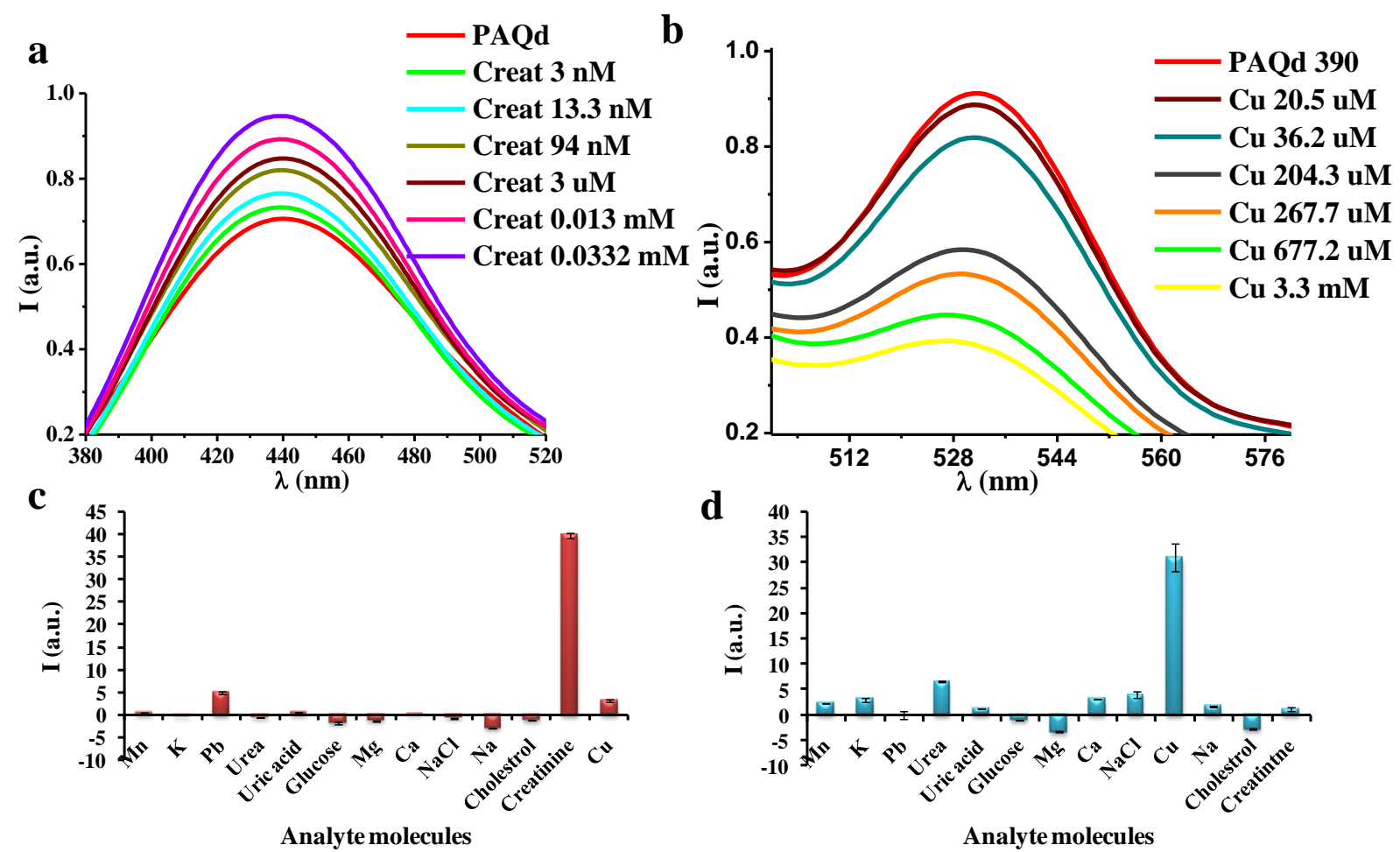

Figure 3. (a) Fluorescence enhancement of PAQd on addition of creatinine $\left(\lambda_{\mathrm{ex}}-290 \mathrm{~nm}\right)(\mathrm{b})$ Fluorescence quenching of PAQd on addition of copper $\left(\lambda_{\mathrm{ex}}-390 \mathrm{~nm}\right)$ (c) Selectivity of creatinine over other analytes and (d) Selectivity of copper over other analytes To demonstrate the multi analyte sensing ability of PAQd, different known concentrations of Creat and $\mathrm{Cu}$ were added to $1 \mathrm{mgmL}^{-1}$ of the same. In the presence of Creat, emission peak c.a. $440 \mathrm{~nm}$ showed concentration dependent fluorescence enhancement when excited at 290 $\mathrm{nm}$. The sensor $\left(1 \mathrm{mgmL}^{-1} \mathrm{PAQd}\right)$ could detect Creat level in the range from $3 \mathrm{nM}$ to 0.003 $\mathrm{mM}$ solution (Figure 3a). Similarly, upon $\mathrm{Cu}$ addition to PAQd (unimilli gram per milli litre), 
the fluorescence quenching of the $531 \mathrm{~nm}$ peak takes place. The copper detection ability ranged from $20.5 \mu \mathrm{M}$ to $3.3 \mathrm{mM}$ (Figure $3 \mathrm{~b}$ ). Thus, in principle, PAQd acts as a turn-on turnoff sensor for creatinine and copper, respectively.

\subsection{Mechanism of sensing}

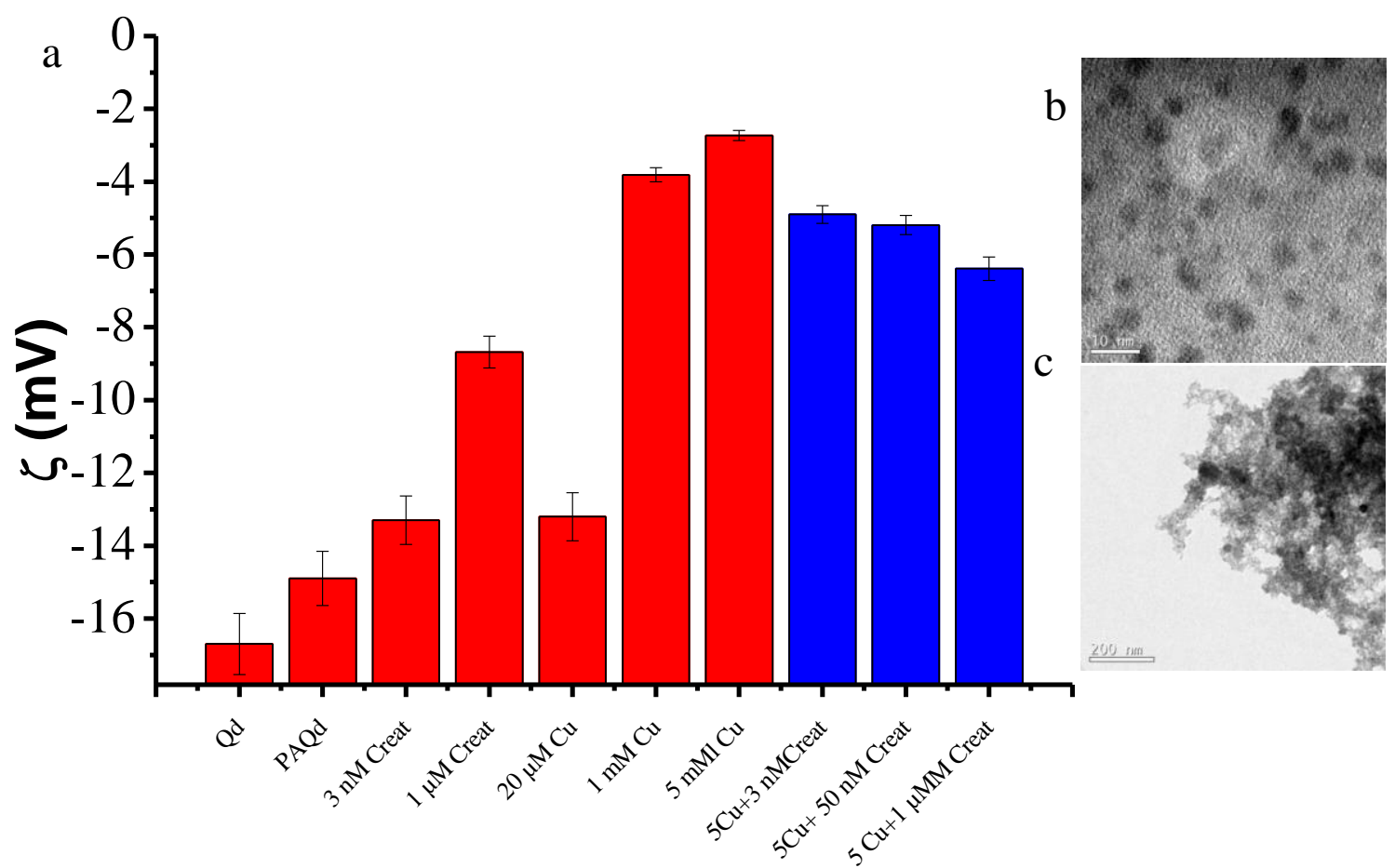

Figure 4. (a) Zeta Potential of Qd, PAQd, PAQd with different concentrations of creatinine (3 nM, $1 \mu \mathrm{M})$, copper $(20 \mu \mathrm{M}, 3 \mathrm{mM}$ and $5 \mathrm{mM}$ ) (Red) and PAQd with $5 \mathrm{mM}$ copper and different concentrations of creatinine ( $1 \mathrm{nM}, 50 \mathrm{nM}$ and $1 \mu \mathrm{M})$ (Blue). (b) TEM image of creatinine incubated PAQd showing well dispersed pattern and c) TEM image of copper incubated PAQd showing the aggregation.

An attempt was made to unravel the mechanism behind the sensing technique. It is observed that the organic layer of PAQd (Figure 1c) disappears on addition of Creat (Figure. 4b).This is because the organic part, picric acid-EDC complex, reacts more strongly with creatinine, reducing the presence of organic layer. Addition of $\mathrm{Cu}$ to PAQd results in increased size of quantum dots (Figure 4c). This is explained based on the decrease in the stability of quantum dots, leading to aggregation. Zeta potential value also supports this; at higher concentrations of $\mathrm{Cu}$, the potential value approaches nearly zero (Figure 4a) and addition of creatinine increases the value. It is the stabilization effect of the large complex molecule in presence of creatinine that prevents Qd from complete aggregation or destabilization. The binding of PA 
to Qd activate the meta nitro group of picric acid leading to the binding of methylene group of creatinine to picric acid. Organic layer of sensor disappears due to the binding of creatinine to Meisenheimer complex, weakening the bond from Qd. The presence of more electron withdrawing groups causes the decrease in zeta potential value. After sensitive region $(\mu \mathrm{M}$ to $\mathrm{mM}$ ), its zeta potential value remains unaltered indicating that only picric acid binding sites are bound to creatinine.

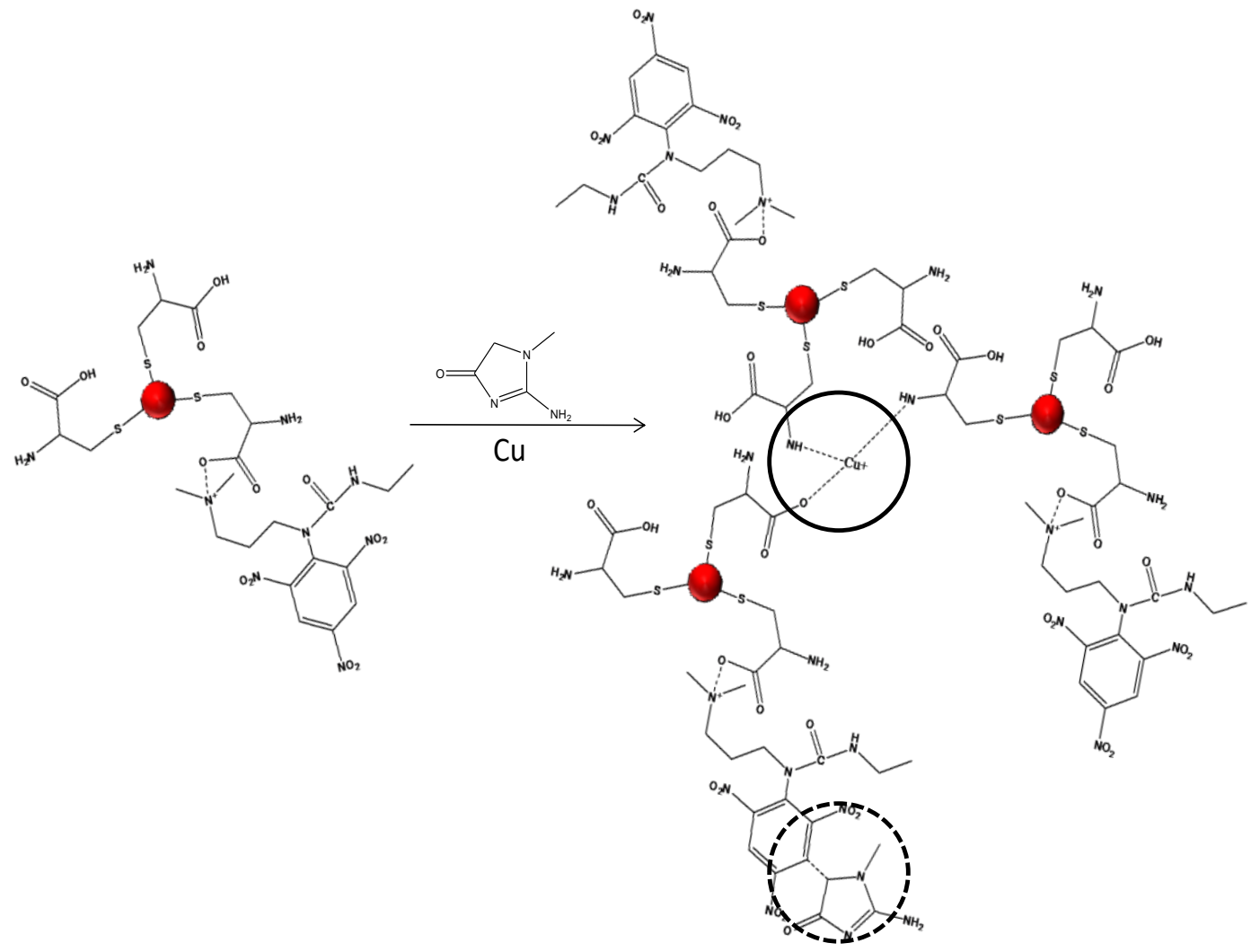

Scheme 2 . Simultaneous detection of copper and creatinine by PAQd. Solid circle represents the complex formation of free cysteine of Qd and copper, dashed circle represents the bond formation of creatinine with picric acid present in PAQd.

Free cysteine present on the surface of the Qd contributes to the detection of $\mathrm{Cu}$ by forming a complex, cuprous bis-cysteine [39] which is responsible for the fluorescence quenching. The complex formation and fluorescence quenching takes place at physiological $\mathrm{pH} 7.4$, and is attributed to inner-filter effects, nonradiative recombination pathways and electron transfer processes $[39,40]$. The electron transfer effects are clear from the observations of size and zeta potential analysis. As the concentration of $\mathrm{Cu}$ increases, size of the probe also increases due to the strong binding of $\mathrm{Cu}$ to cysteine leading to the clustering of $\mathrm{Qd}$ followed by 
increase in the size. The zeta potential value approaches zero upon addition of higher amount of $\mathrm{Cu}$, but never reaches zero. This is because of the presence of picric acid EDC complex over Qd (Figure 4a). Scheme 2 represents the expected mechanism of sensing.

\subsection{Selectivity of sensing}

On confirming the efficiency of PAQd for simultaneous sensing of Creat and $\mathrm{Cu}$, selectivity against other heavy metals and small biomolecules such as $\mathrm{Pb}, \mathrm{Mg}, \mathrm{Mn}, \mathrm{Ca}$, urea and uric acid were checked. The changes in the emission peaks were found to be specific to Creat and $\mathrm{Cu}$ (Figure 3c, d) without any interference from the presence of these metals or molecules. The simultaneous detection capacity of the sensor was also monitored from the zeta potential values and selectivity studies. For this, creatinine was added to copper enriched PAQd which is susceptible for aggregation (Figure 4a), and the results were monitored. It is found that there is no interference of creatinine on $\mathrm{Cu}$ detection and vice versa.

\subsection{Detection of copper and creatinine from blood serum}

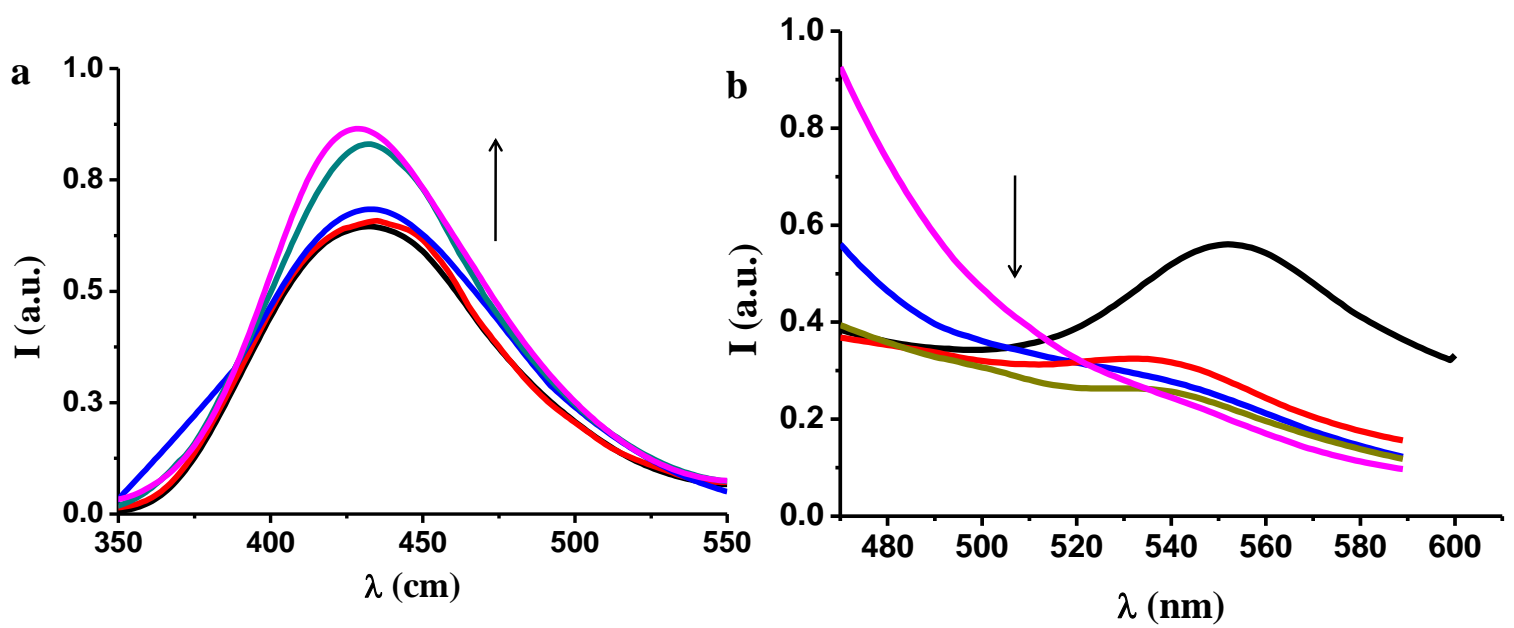

Figure 5. (a) Detection of creatinine in blood serum using PAQd [Black- PAQd, Red to Pinkincrease in creatinine concentration; arrow indicate the increase in fluorescence intensity] (b) detection of copper in blood serum using PAQd [Black- PAQd, Red to Pink- increase in copper concentration; arrow indicate the decrease in fluorescence intensity]

The efficacy of the sensor was checked in blood serum as an initial step to extend the results to clinical use. For this, copper and creatinine were added to the human blood serum containing $1 \mathrm{mg} / \mathrm{ml}$ of sensor, and checked the photoluminescence after 2 min. incubation. The results resembled to that of aqueous medium detection. The emission peak at $440 \mathrm{~nm}$ increased with increasing concentration of creatinine (Figure 5a) and the emission intensity at 
$531 \mathrm{~nm}$ decreased (Figure 5b) with increasing concentration of $\mathrm{Cu}$ proving the efficiency of the simultaneous determination of $\mathrm{Cu}$ and creatinine from human blood serum.

\subsection{Detection of copper and creatinine in the cellular environment}

Efficacy of this sensor was also checked in cellular environment since both Creatinine and $\mathrm{Cu}$ are playing vital role in metabolic activity, and regulate and influence the blood analyte levels. Before performing the cellular study, the cytotoxicity of Qd and PAQd was assessed using MTT assay in two different cells viz. in L929 mouse fibro blast and MDA MB 231 human breast cancer cells. The cells were viable (Figure.S6) for different concentrations of PAQd upto $1 \mathrm{mgmL}^{-1}$ at $72 \mathrm{~h}$. Later, sensing efficacy of PAQd towards Creat and $\mathrm{Cu}$ was checked on the above two cells.

Because analytes of interest could be present both inside and outside the cells, the extracellular and intracellular sensing efficacy of the sensor was checked. For intracellular creatinine sensing, cells were incubated with Creat for 30 minutes and washed with PBS and then incubated with PAQd for 3h. Similarly, intracellular $\mathrm{Cu}$ was also evaluated. The timing of incubation was based on the fact that copper and creatinine being small biomolecules, are expected to get internalized by the cells more quickly compared to complex nanostructures. Enhanced fluorescence for creatinine and fluorescence quenching for $\mathrm{Cu}$ was observed under fluorescence microscopy. For extra cellular Creat sensing, initially the sensor PAQd was incubated with the cells for 30 minutes followed by incubation of creatinine for 15 minutes. The same procedure was used for extracellular sensing of copper. Fluorescence enhancement or quenching with creatinine and $\mathrm{Cu}$ was observed for L929 cells whereas MDAMB cells did not show any significant change. The reason for this observed difference between two cell lines is due to the difference in the environmental conditions of cancer cells and normal cells. In the case of intracellular sensing, when the analyte was incubated first, due to ion channel effect, analyte molecules get trapped into the cell. However, in the case of extracellular sensing, when PAQd is added first, the cancer cells would have blocked the binding site of the probe, reducing its functionality to interact with the analyte molecules, added subsequently (FigureS7\&Figure S8).

\subsection{Mobile App based sensing using paper strip sensors: validation with clinical results}

To extend the findings to the clinical application, paper strip based sensor was developed to check the levels of inherent creatinine present in human serum from unknown samples $(n=4)$ 
(Figure S9). On addition of a series of concentrations of creatinine to the sensor strips, pixel intensity from the mobile camera images of $254 \mathrm{~nm}$ irradiated strips was plotted and calibrated to estimate unknown sample concentration (Figure S10). Later, the results were compared with blood creatinine value of the same individuals from their previous medical records (Figure. S11). The creatinine values obtained with PAQd sensor were 0.7, 0.8, 0.9, and 1.2, and the clinical values recorded were1.1, 0.8,0.9, and 0.9.In two cases (2nd\& $3 \mathrm{rd}$ ), the PAQd data were comparable with the clinically used Jaffe reaction result with negligible variation. However, the $1^{\text {st }}$ and $3^{\text {rd }}$ cases showed slightly higher levels as per their records. On interacting with them to find the reason for the change, it was found that both these individuals were on medication for other treatments, when the blood for PAQd sensing was drawn. We presume that the effect of the medicine would have been the reason for the reduced level with PAQd sensor. More detailed study with a higher number of patient samples have to be tested and validated in future.

\section{Conclusions}

To summarize, a quantum dot based nanosensor for simultaneous detection of copper and creatinine at normal conditions of $\mathrm{pH}$ and temperature was synthesized and the mechanism of its formation is explained. The turn on turn off sensor was highly efficient in detecting copper and creatinine in blood serum. It could also detect the intracellular and extracellular $\mathrm{Cu}$ and creatinine in cellular environment. The selectivity of the developed sensor was also proven towards the analytes considered. A simple, mobile phone based quantification of creatinine from human blood has also been proven using paper strips of the quantum dot based sensor. Synthesis of such a sensor for detection of multianalytes will open up new horizon in the biomedical detection systems. Using such systems, healthcare industry can provide cheap and multi analyte detection kit which can detect different analytes on single draw of blood, quickly and cost effectively. Future works on this context is expected to be very promising.

\section{Acknowledgements}

The authors acknowledge the support received from Dr K.K Maiti and his team from CSIRNIIST, Trivandrum and Dr Reji Varghese and his team from IISER, Trivandrum for the TEM studies. Authors also thank Dr. Luxmi Varma R and her team from CSIR-NIIST, Trivandrum for NMR analysis support. Support received from Sreejalekshmi K.G. and Mr. Rakesh from IIST, Trivandrum for fluorescence life time studies is acknowledged. 


\section{Notes}

The work utilized the funds under the project BT/PR14763/NNT/28/961/2015 by Department of Biotechnology, Government of India for meeting the expenses in connection with consumables and testing charges.

\section{References}

[1] C.Wu, M. Kyaw, K.Oo, X. Fan, Highly Sensitive Multiplexed Heavy Metal Detection Using Quantum-Dot-Labeled DNAzymes. ACS Nano, 4(2010), 5897-5904. https://doi.org/10.1021/nn1021988.

[2] W.Wu, Z.Sun, Y. Zhang, J.Xu, H.Yu, X. Liu, Q. Wang, A Multifunctional Nanosensor Based on Silica Nanoparticles and Biological Applications in Living Cells. Chem. Commun., 48 ( 2012) 11017-11019. https://doi.org/10.1039/c2cc36686j

[3] H. Komatsu, T. Miki, D.Citterio, T. Kubota, Y.Shindo, Y. Kitamura, K.Oka, K.Suzuki, Single Molecular Multianalyte $\left(\mathrm{Ca}^{2+}, \mathrm{Mg}^{2+}\right)$ Fluorescent Probe and Applications to Bioimaging. J. Am. Chem. Soc., , 127(2005), 10798-10799. https://doi.org/10.1021/ja0528228

[4] M.J. Ruedas-rama, E.A.H. Hall, Analytical Nanosphere Sensors Using Quantum Dot Enzyme Conjugates for Urea and Creatinine. Anal. Chem., 82(2010), 9043-9049. https://doi.org/10.1117/12.842640

[5] A. Sugunan, C.Thanachayanont, J. Dutta, J.G. Hilborn, Heavy-Metal Ion Sensors Using Chitosan-Capped Gold Nanoparticles. Sci. Technol. Adv. Mater., 335(2005), 335-340. https://doi.org/10.1016/j.stam.2005.03.007

[6] W Wang, Q Wen, Y Zhang, X Fei, Y Li,a Q Yang, X Xu, Simple naphthalimidebased fluorescent sensor for highly sensitive and selective detection of $\mathrm{Cd}^{2+}$ and $\mathrm{Cu}^{2+}$ in aqueous solution and living cells. Dalton Trans., 42(2013),1827-1833. https://doi.org/10.1039/C2DT32279J 
[7] A Mondal, A. R. Chowdhury, SBhuyan, S. K. Mukhopadhyay, P. Banerjee, A simple urea-based multianalyte and multichannel chemosensor for the selective detection of $\mathrm{F}^{-}$, $\mathrm{Hg}^{2+}$ and $\mathrm{Cu}^{2+}$ in solution and cells and the extraction of $\mathrm{Hg}^{2+}$ and $\mathrm{Cu}^{2+}$ from real water sources: a logic gate mimic ensemble. Dalton Trans., 48(2019), 4375-4386. https://doi.org/10.1039/C8DT05097J

[8] S. Ghosh, AGanguly, Md R Uddin, S Mandal, Md. A. Alam N Guchhait, Dual mode selective chemosensor for copper and fluoride ions: a fluorometric, colorimetric and theoretical investigation. Dalton Trans., 45(2016), 11042-11051. https://doi.org/10.1039/C6DT00968A

[9] L. V.Nair, Y.Nagaoka, T.Maekawa,D.Sakthikumar, R.S. Jayasree, Quantum Dot Tailored to Single Wall Carbon Nanotubes: A Multifunctional Hybrid Nanoconstruct for Cellular Imaging and Targeted Photothermal Therapy. Small, 10(2014), 2771-2775. https://doi.org/10.1002/smll.201400418

[10] L. V. Nair, D.S. Philips, R.S. Jayasree, A. Ajayaghosh, A Near-Infrared Fluorescent Nanosensor ( AuC @ Urease ) for the Selective Detection of Blood Urea. Small, 9(2013), 2673-2677. https://doi.org/10.1002/smll.201300213

[11] L. V. Nair, R. V. Nair, R.S. Jayasree, An insight into the optical properties of a sub nanosize glutathione stabilized gold cluster, Dalton Trans.,45( 2016), 11286-11291. https://doi.org/10.1039/c6dt01753c

[12] L. V. Nair, R. V. Nair, S.J. Shenoy, A. Thekkuveettil, R.S. Jayasree, Blood brain barrier permeable gold nanocluster for targeted brain imaging and therapy: an in vitro and in vivo study, J. Mater. Chem. B., 5(2017), 8314-8321. https://doi.org/10.1039/c7tb02247f

[13] L. V. Nair, S.S. Nazeer, R.S. Jayasree, A. Ajayaghosh, Fluorescence Imaging Assisted Photodynamic Therapy Using Photosensitizer-Linked Gold Quantum Clusters. ACS Nano, 9(2015), 5825-5832. https://doi.org/10.1021/acsnano.5b00406 
[14] R. V. Nair, H. Santhakumar, R.S. Jayasree, Gold Nanorods Decorated with a Cancer

Drug for Multimodal Imaging and Therapy. Faraday Discuss., 207(2018), 423-435.

https://doi.org/10.1039/c7fd00185a

[15] H. Santhakumar, R. V. Nair, D.S. Philips, S.J. Shenoy, R.S. Jayasree, Real Time

Imaging and Dynamics of Hippocampal Zn 2 + under Epileptic Condition Using a

Ratiometric Fluorescent Probe. Sci. Rep., 8(2018), 1-11. https://doi.org/10.1038/s41598-018-

$\underline{27029-5}$

[16] C. V. Durgadas, L. V.Nair, C.P. Sharma, K.Sreenivasan, Chemical Sensing of Lead Ions Using Glutathione Mediated End to End Assembled Gold Nanorod Chains. Sensors Actuators B. Chem., 156(2011), 791-797. https://doi.org/10.1016/j.snb.2011.02.040

[17] A.Saraswathy, S.S. Nazeer, M. Jeevan, N. Nimi, S.Arumugam, V.S. Harikrishnan, P.R.. Varma, R.S. Jayasree, Citrate Coated Iron Oxide Nanoparticles with Enhanced Relaxivity for in Vivo Magnetic Resonance Imaging of Liver Fibrosis A. Colloids Surfaces B Biointerfaces, 117(2014), 3-5. https://doi.org/10.1016/j.colsurfb.2014.02.034

[18] A.Saraswathy, S.S. Nazeer, N. Nimi, S.Arumugam, S.J. Shenoy, R.S. Jayasree, Synthesis and Characterization of Dextran Stabilized Superparamagnetic Iron Oxide Nanoparticles for in Vivo MR Imaging of Liver Fibrosis. Carbohydr. Polym., 101(2014), 760-768. https://doi.org/10.1016/j.carbpol.2013.10.015

[19] W. Wei, X. Zhang, S. Zhang,G. Wei, Z. Su, Biomedical and bioactive engineered nanomaterials for targeted tumor photothermal therapy: A review, Mater. Sci. Eng. C, 104(2019), 109891. https://doi.org/10.1016/j.msec.2019.109891

[20]M.Norouzi,S.Yasamineh,M.Montazeri,M.Dadashpour,R.Sheervalilou,M. Abasi,Y. Pilehvar-Soltanahmadi, Recent advances on nanomaterials-based fluorimetric approaches for 
microRNAs detection, Mater. Sci. Eng. C, 104(2019), 110007.

https://doi.org/10.1016/j.msec.2019.110007

[21] R.Eivazzadeh-Keihan, K. K. Chenab,R. Taheri-Ledari,J. Mosafer, S.M. Hashemi, A. Mokhtarzadeh, A. Maleki, Michael R.Hamblin, Recent advances in the application of mesoporous silica-based nanomaterials for bone tissue engineering, Mater. Sci. Eng. C, (2019), 110267. https://doi.org/10.1016/j.msec.2019.110267

[22] S.H. Kreisman, J. V. Hennessey, Consistent Reversible Elevations of Serum Creatinine Levels in Severe Hypothyroidism. Arch Intern Med., 159(1999), 79-82.

[23] J.P. Matts, J.N. Karnegis, C.T. Campos, L.L. Fitch, J.W. Johnson, H. Buchwald Serum Creatinine as an Independent Predictor of Coronary Heart Disease Mortality in Normotensive Survivors of Myocardial. Fam Pr., 36(1993), 497-503.

[24] J. Du, B. Zhu, W.R. Leow, S.Chen, T.C. Sum, X.Peng, X.Chen, Colorimetric Detection of Creatinine Based on Plasmonic Nanoparticles via Synergistic Coordination Chemistry. Small, 11(2015),4104-4110. https://doi.org/10.1002/smll.201403369

[25] I.R. Reid, S.M.Bristow, M.J. Bolland, Calcium and Cardiovascular Disease. Endocrinol Metab, 32(2017), , 339-349. https://doi.org/10.3803/EnM.2017.32.3.339

[26] R.Uauy, M.Olivares, M.Gonzalez, Essentiality of Copper in Humans Essentiality of Copper in Humans. Am J Clin Nutr, 67(1998), 952S-9S.

https://doi.org/10.1093/ajen/67.5.952S

[27] V.N. Mehta, M.A. Kumar, S.K. Kailasa, Colorimetric Detection of Copper in Water Samples Using Dopamine Dithiocarbamate-Functionalized Au Nanoparticles. Ind. Eng. Chem, 52(2013),4414-4420. https://doi.org/10.1021/ie302651f 
[28] T. Lou, L.Chen, Z.Chen, Y. Wang, L.Chen, J. Li, Colorimetric Detection of Trace Copper Ions Based on Catalytic Leaching of Silver-Coated Gold Nanoparticles. ACS Appl. Mater. Interface, 3(2011),4215-4220. https://doi.org/10.1021/am2008486

[29] X.Qiu, S. Han, Y. Hu, B. Sun, Ratiometric Fluorescent Nanosensors for Copper ( II ) Based on Bis ( Rhodamine ) -Derived PMOs with J-Type Aggregates. Chem. Eur. J., 21(2015), 4126-4132. https://doi.org/10.1002/chem.201406143

[30] Y.Song, K. Qu, C. Xu, J. Ren, X. Qu, Visual and Quantitative Detection of Copper Ions Using Magnetic Silica Nanoparticles Clicked on Multiwalled Carbon Nanotubes. Chem. Commun., 46(2010), , 6572-6574. https://doi.org/10.1039/c0cc01593h

[31] S. Kacmaz, K. Ertekin, D. Mercan, O. Oter, E. Cetinkaya, E. Celik, Spectrochimica Acta Part A : Molecular and Biomolecular Spectroscopy An Ultra Sensitive Fluorescent Nanosensor for Detection of Ionic Copper. Spectrochim. ACTA PART A Mol. Biomol. Spectrosc., 135(2015), 551-559. https://doi.org/10.1016/j.saa.2014.07.056

[32] H. Du, R. Chen, J. Du, J.Fan, X. Peng, Gold Nanoparticle-Based Colorimetric Recognition of Creatinine with Good Selectivity and Sensitivity. Ind. Eng. Chem. Res, 55(2016), 12334-12340. https://doi.org/10.1021/acs.iecr.6b03433

[33] S. Mohammadi, G. Khayatian, Highly Selective and Sensitive Photometric Creatinine Assay Using Silver Nanoparticles. Microchim Acta, 182(2015), 1379-1386. https://doi.org/10.1007/s00604-015-1460-5

[34] P. Jose, H.Skali, N. Anavekar, C.Tomson, H.M.Krumholz, J.L.Rouleau, L.Moye, M.A.Pfeffer, S.D. Solomon, Increase in Creatinine and Cardiovascular Risk in Patients with Systolic Dysfunction after Myocardial Infarction. J Am Soc Nephrol, 17(2006), 2886-2891. https://doi.org/10.1681/ASN.2006010063 
[35] A. Lee, J. Chin, O. K. Park, H. Chung, J. W. Kim, S. Y. Yoon, K. Park, A novel near-infrared fluorescence chemosensor for copper ion detection using click ligation and energy transfer. Chem. Commun., 2013,49, 5969-5971.

https://doi.org/10.1039/C3CC42059K

[36] J Liang, M Qin, R Xu, X Gao, Y Shen, Q Xu, Y Cao, W Wang, A genetically encoded copper(i) sensor based on engineered structural distortion of EGFP. Chem.

Commun., 2012,48, 3890-3892. https://doi.org/10.1039/C2CC30531C

[37] G.R. Amiri, S.Fatahian, S.Mahmoudi, Preparation and Optical Properties Assessment of CdSe Quantum Dots. Mater. Sci. Appl., 4(2013), 134-137.

https://doi.org/10.4236/msa.2013.42015

[38] R.O. Al-kaysi, D.Creed, E.J. Valente, Meisenheimer Complex from Picric Acid and Diisopropylcarbodiimide. J. Chem. Crystallogr., 34(2004),685-692. https://doi.org/10.1023/B:JOCC.0000047644.13643.c3

[39] L.Pecci, G.Montefoschi, G.Musci, D.Cavallini, Novel findings on the copper catalysed oxidation of cysteine. Amino Acids, 13(1997) 355-367. https://doi.org/10.1007/BF01372599

[40] M.F.Frasco, N.Chaniotakis, Semiconductor Quantum Dots in Chemical Sensors and Biosensors. sensors, 9(2009), 7266-7286. https://doi.org/10.3390/s90907266 